\title{
Inhalt des 4. Bandes
}

Heft 1

R. Adams

Abstufung von Schlepperklassen. . . . . . . . . . . . . . . . . . . 3

K. BAGANZ

Spannungs- und Verdichtungsmessungen im Boden bei verschiedenen Fahrgeschwindigkeiten. . . . . . . . . . . . . . . . . . . . . . . . 35

G. KRUPP

Messungen zur Qualität der Bodenbearbeitung unmittelbar nach Durchgang des Gerätes. . . . . . . . . . . . . . . . . . . . . . . . . . . . 47

H. TISCHLER

Technisch getrennte Ernte von Zuckerrüben und -blatt in einer Phase . . . $\quad 57$

\section{Heft 2}

W. BALKIN

Die Siebleistung horizontal schwingender Plansiebe in Abhängigkeit von

Siebneigung, Schwingungszahl und Schwingungsweite . . . . . . . 109

L. KUNATH

Dichte- und Ertragsmessungen in Schlepperfahrspuren . . . . . . . . . 137

G. KRUPP

Über den Schlupf am treibenden Drillmaschinenrad und dessen Wirkung

auf die Bestandsdichte der ausgedrillten Kultur . . . . . . . . . . . . 145

W. MALTRY

Elektrische Federwaage EFW 10/61 mit Einkanalverstärker TF I/61 . . . 151

G. KÜHN

Teilautomatisches Gerät für die statistische Auswertung von Meßschrieben 167

\section{Heft 3}

M. TSCHIERSCHKE

Das Verhalten fließfähiger Futtermischungen durch horizontale Rohr-

leitungen . . . . . . . . . . . . . . . . . . . . . 177

R. ADAMS

Ermittlung des Energiebedarfes für die Feldwirtschaft . . . . . . . . . 219

G. KÜHN

Bodenantriebsräder für Anbaumaschinen. . . . . . . . . . . . . . . 243

\section{Autorenverzeichnis}

Scite Seite

Adams, R. . . . . . . . . . . 3,219 KUnath, L. . . . . . . . . . . 137

BaGanz, K. . . . . . . . . . . . . 35 Maltry, W. . . . . . . . . . . 151

Balkin, W. . . . . . . . . . . . . . 109 Trschler, H. . . . . . . . . . . . $\quad .57$

KRUPP, G. . . . . . . . . . . . 47,145 TschinRSCHKE, M. . . . . . . . $\quad .177$

K̈̈HN, G. . . . . . . . . . 167,243 